\title{
On the calculus of Dirac delta function with some applications in classical electrodynamics
}

\author{
Milan S. Kovačević, Miroslav R. Jovanović and Marko M. Milošević \\ University of Kragujevac, Faculty of Science, Department of Physics, Serbia.
}

Received 26 January 2021; accepted 16 March 2021

\begin{abstract}
The Dirac delta function is a concept that is useful throughout physics as a standard mathematical tool that appears repeatedly in the undergraduate physics curriculum including electrodynamics, optics, and quantum mechanics. Our analysis was guided by an analytical framework focusing on how students activate, construct, execute, and reflec on the Dirac delta function in the context of classical electrodynamics problems solving. The applications of Dirac delta function to fin the charge density associated with a point charge as well as electrostatic point dipole field for more advanced situations to describe the charge density of hydrogen atom were presented.
\end{abstract}

Keywords: Delta function; point charge; electric dipole; magnetic current.

PACS: 41.20.-q; 41.20.Cv; 41.20.Gz

DOI: https://doi.org/10.31349/RevMexFisE.18.020205

\section{Introduction}

The delta functions appeared in the early days of $19^{\text {th }}$ century, in works of the Poission (1815), Fourier (1822) and Cauchy (1823) [1]. Subsequently Heaviside (1883) and Kirchoff (1891) gave the firs mathematical definition of the delta functions. Kirchoff was concerned with the fundamental solution of the three-dimensional wave equation, while Heaviside introduced the function in his "Operational Calculus". He pointed that $\delta(x)$ could be regarded as the derivation of the Heaviside or unit "step function" $\theta(x)$, define as unity for $x>0$ and zero for $x<0$. After Heaviside, the delta function was freely used -in particular, in connection with Laplace transforms, especially by electrical engineers (e.g. Van der Pol (1928))-. Dirac (1926, 1930) introduced the delta function in his classic and fundamental work on the quantum mechanics, essentially as the continuous analogue of the Kronecker delta. He also wrote down, a list of its important properties [2,3]. The uses of the delta function became more and more common after the appearance of Dirac's work. Other singular functions also made their appearance, as early version of quantum fiel theory began to take shape in the words of physicists such as Jordan, Pauli and Heinsenberg. Around the same time, mathematicians began attempts to defin such singular quantities in a rigorous manner.

The delta function and other such singular objects were recognized to be what are called generalized functions or distribution, rather than function in the usual mathematical sense. These were introduced in quantum mechanics by Dirac [3] who did not, however, fin them a source of lack of rigor in the theory. The mathematical justificatio of the use of the Dirac delta has been given by Schwartz (1950) in his
Theory des Distributions [4]. A much more accessible version of the theory based on the concept of generalized functions define by means of sequences was developed by Temple, and a very readable treatment is given by Lighthill [5].

Dirac delta functions provide a convenient way to describe the singular nature of certain distributions, which are widely used in electrodynamics, quantum optics and other areas of quantum physics. How to deal in a consistent and systematic manner with singularities arising at the origin of a polar coordinates system is a recurring question in the teaching of classical electrodynamics $[6,7,9]$ as well as in research [10-12]. In the paper [13] an analytical framework describing the use of mathematical tools in physics problem solving to structure investigation and analysis of student diffi culties with $\delta$ functions was given. The use of generalized delta function to derive the Glauber-Sudarshan P-function for a Schrödinger cat state in a surprisingly simple form is presented in [14]. Aside from their potential applications in classical electromagnetism and quantum optics, these results provide insight into the ability of the diagonal P-function to describe density operators with off-diagonal elements. The electric or magnetic fiel of an ideal dipole is known to have a Dirac delta function at the origin. In Ref. [15] it is shown that the divergence of a singular vector fiel can contain a derivative of a Dirac delta function even if the fiel itself does not contain a delta function.

In undergraduate curriculum, Dirac delta function is used in a variety of contexts throughout physics curriculum. However, we have observed consistent student difficultie using the delta function. This paper presents various ways of defin ing and introducing Dirac delta function including its application in solving some problems in electrodynamics. 


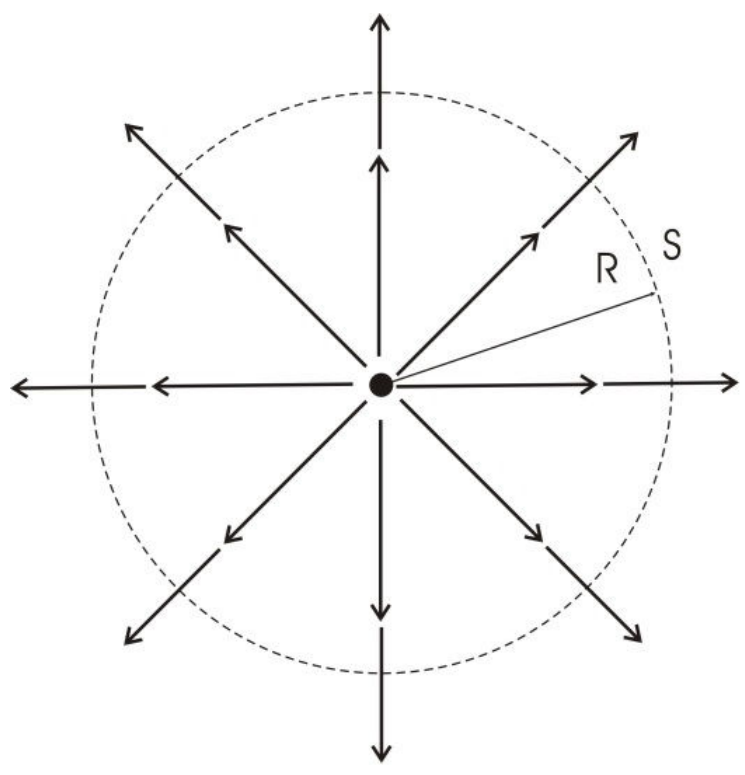

FIGURE 1 . The vector function $\mathbf{v}$ directed radially outward.

\section{Why does the Dirac delta function appear in physical problems?}

Consider the vector function

$$
\mathbf{v}=\frac{1}{r^{2}} \mathbf{r}_{0}
$$

where $\mathbf{r}_{0}$ is unit vector in radial direction. The function $\mathbf{v}$ is directed radially outward (Fig. 1); if ever there was a function that ought to have a large divergence, this is it.

Now we calculate the divergence of vector function (1); we get precisely zero:

$$
\begin{aligned}
\nabla \cdot \mathbf{v} & =\frac{1}{r^{2}} \frac{\partial}{\partial r}\left(\frac{1}{r^{2}} v_{r}\right)=\frac{1}{r^{2}} \frac{\partial}{\partial r}\left(\frac{1}{r^{2}} r^{2}\right) \\
& =\frac{1}{r^{2}} \frac{\partial}{\partial r}(1)=0 .
\end{aligned}
$$

The result in Eq. (2) may come as a surprise; this is a paradox. The divergence $\nabla \cdot \mathbf{v}$ is a measure of how much the vector $\mathbf{v}$ spreads out (diverges) from the point in question. For example, the vector function in Fig. 1 has a large (positive) divergence. It should be understood that is a function -there is a different vector associated to every point in space-. Now, apply the fundamental theorem of divergence using the function in Eq. (1). The fundamental theorem of divergence states that:

$$
\int_{V}(\nabla \cdot \mathbf{v}) d \tau=\oint_{S} \nabla \cdot d \boldsymbol{\sigma}
$$

Suppose we integrate over a sphere of radius $R$, centered at the origin; the surface integral is

$$
\begin{aligned}
\oint_{S} \nabla \cdot d \boldsymbol{\sigma} & =\int\left(\frac{1}{R^{2}} \mathbf{r}_{0}\right)\left(R^{2} \sin \theta d \theta d \phi \mathbf{r}_{0}\right) \\
& =\int_{0}^{\pi} \sin \theta d \theta \int_{0}^{2 \pi} d \phi=4 \pi .
\end{aligned}
$$

But the volume integral $\int_{V}(\nabla \cdot \mathbf{v}) d \tau$ on the left hand of Eq. (3) gives the zero. What's going on here? The source of the problem is the point $r=0$ where $\mathbf{r}$ blows up (Fig. 1). It should be noticed that the surface integral in Eq. (4) is independent of $R$; if the divergence theorem is right, we should get $\int_{V}(\nabla \cdot \mathbf{v}) d \tau=4 \pi$ for any sphere centered at the origin, no matter how small. Hence $\nabla \cdot \mathbf{v}$ has a bizarre property that it vanishes everywhere except at one point, and yet its integral over any volume containing that point is $4 \pi$. There is no ordinary mathematical function behavior like that. On the other hand, a physical example does come to mind: if $\mathbf{v}$ represent the $\mathrm{fl} \mathrm{w}$ of and incompressible fluid then the flu of $\mathbf{v}$, the right side of Eq. (3), is the total amount of flui passing out through the surface, per unit time. Now, the divergence measures the 'spreading out' of the vectors from a point -a place of high divergence is like a 'faucet' pouring out liquid-. We also have the similar problem of a point particle: consider the basic problem of electrostatics. Given a static charge density $\rho(\mathbf{r})$ in free space, what is the corresponding electrostatic potential $\phi(\mathbf{r})$ at any arbitrary point $\mathbf{r}=(x, y, z)$ ? From Maxwell's equations, we know that $\phi$ satisfie Poisson's equation [17], namely,

$$
\nabla^{2} \phi(\mathbf{r})=-\frac{\rho(\mathbf{r})}{\varepsilon_{0}},
$$

where $\varepsilon_{0}$ is the permittivity of the vacuum. What does one do in the case of a point charge $q$ located at the same point $\mathbf{r}_{0}=\left(x_{0}, y_{0}, z_{0}\right)$ ? A point charge is an idealization in which a finit amount of charge $q$ is supposed to be placed into zero volume. The charge density must therefore be infinit at the point $\mathbf{r}_{0}$, and zero elsewhere. These paradoxes can be overcome by introducing a mathematical object known to physicist as the Dirac delta function. The delta function comes to our aid: we may write, in this case,

$$
\rho(\mathbf{r})=q \delta^{(3)}\left(\mathbf{r}-\mathbf{r}_{0}\right),
$$

where $\delta^{(3)}$ is the three-dimensional delta function. It arises in many branches of theoretical physics, especially in quantum mechanics and electrodynamics.

\section{Dirac delta function: properties and repre- sentations}

The delta 'function' $\delta\left(x-x^{\prime}\right)$ is such that for any other function $f: R \rightarrow R$ :

$$
\int_{-\infty}^{+\infty} f(x) \delta\left(x-x^{\prime}\right) d x=f\left(x^{\prime}\right) .
$$


A special case of this is when $f$ identically 1 , giving is

$$
\delta\left(x-x^{\prime}\right) d x=1 .
$$

The delta function is best thought of as a functional, something that takes a function to a value. In the above examples, the delta 'plucks out' the value of $f$ at $x$ from under an integral. The one-dimensional Dirac delta function $\delta\left(x-x^{\prime}\right)$ can be pictured as an infinitel high infinitesimall narrow 'spike' with area 1. That is to say:

$$
\begin{aligned}
& \delta\left(x-x^{\prime}\right)=\left\{\begin{array}{l}
0, \quad \text { if } x \neq x^{\prime} \\
\infty, \quad \text { if } x=x^{\prime}
\end{array}\right. \\
& \text { with } \int_{-\infty}^{+\infty} \delta\left(x-x^{\prime}\right) d x^{\prime}=1 .
\end{aligned}
$$

In the point $x^{\prime}=0$, we have

$$
\begin{aligned}
& \delta(x)=\left\{\begin{array}{cc}
0, & \text { if } \quad x \neq 0 \\
\infty, & \text { if } \quad x=0
\end{array}\right. \\
& \text { and } \int_{-\infty}^{+\infty} \delta(x) d x=1 .
\end{aligned}
$$

Dirac delta function is that it is not a function at all since its value is not finit at $x=0$. In mathematical literature it is known as a generalized function or distribution.

In physics, the function $\delta^{(3)}(\mathbf{r})$ (the three-dimensional Dirac delta function) is define by

$$
\delta^{(3)}(\mathbf{r})=\delta(x) \delta(y) \delta(z)
$$

with the following properties: $\delta^{(3)}(\mathbf{r})=0$ everywhere except at the singular point $(0,0,0)$ where it blows up. Its volume integral is 1 :

$$
\begin{aligned}
& \int_{\text {all space }} \delta^{(3)}(\mathbf{r}) d \tau \\
= & \int_{-\infty}^{+\infty} \int_{-\infty}^{+\infty} \int_{-\infty}^{+\infty} \delta(x) \delta(y) \delta(z) d x d y d z=1 .
\end{aligned}
$$

By the changing variables to $\mathbf{r}-\mathbf{r}^{\prime}$ in following integral, it is easy to see using (7) that

$$
\int_{\text {all space }} f(\mathbf{r}) \delta^{(3)}\left(\mathbf{r}-\mathbf{r}^{\prime}\right) d \tau=f\left(\mathbf{r}^{\prime}\right) .
$$

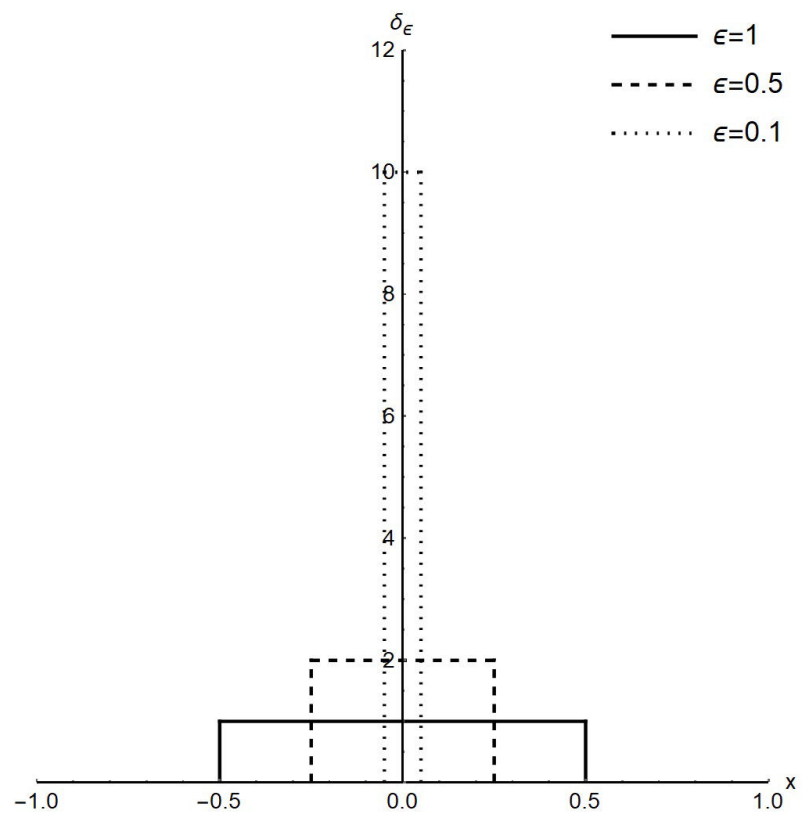

FIGURE 2. The function $\delta_{\varepsilon}(x)$ for $\varepsilon=1.0,0.5$ and 0.1 .

As in the one-dimensional case, integration with $\delta_{\varepsilon}(x)$ picks out the value of the function $f$ at the location of the spike.

\section{On the calculus of Dirac delta function}

In this section we have used the Wolfram Mathematica to present a few rigorous approaches of the Dirac delta function. The simplest set of function with the proper behavior is the set function $\delta_{\varepsilon}(x)$ define by

$$
\delta_{\varepsilon}(x)=\left\{\begin{array}{c}
1 / \varepsilon \text { for }|x| \leq \varepsilon / 2 \\
0 \text { for }|x| \leq \varepsilon / 2
\end{array} .\right.
$$

In the Fig. 2 the function $\delta_{\varepsilon}(x)$ is pictured.

Clearly, we have $\lim _{\varepsilon \rightarrow 0} \delta_{\varepsilon}(x)=0$ for all $x \neq 0$, and $\int_{-\infty}^{+\infty} \delta_{\varepsilon}(x) d x=1$ independent on $\varepsilon$. The function $\delta_{\varepsilon}(x)$ is define for all $\varepsilon \neq 0$, and we have $\lim _{\varepsilon \rightarrow 0} \int_{-\infty}^{+\infty} \delta_{\varepsilon}(x) d x=$ 1.

The second representation of Dirac delta function comes from the sequence of Gaussian functions define by

$$
\delta_{a}(x)=\frac{1}{\alpha \sqrt{\pi}} e^{-x^{2} / \alpha^{2}} .
$$

Note that $\lim _{\alpha \rightarrow 0} \delta_{\alpha}(x)=0$ for all $x \neq 0$. And using the well-known results $\int_{-\infty}^{+\infty} e^{-\alpha^{2}} d x=\sqrt{\pi / \alpha}$ we see that $\int_{-\infty}^{+\infty} \delta_{\alpha}(x) d x=1$ is independent of $\alpha$. In Fig. 3, the function $\delta_{\alpha}(x)$ is plotted for three different values of $\alpha$.

The third useful representation of the Dirac delta function is

$$
\delta(x)=\lim _{\varepsilon \rightarrow 0} \delta_{\varepsilon}(x)=\lim _{\varepsilon \rightarrow 0} \frac{1}{\pi} \frac{\varepsilon}{x^{2}+\varepsilon^{2}} .
$$




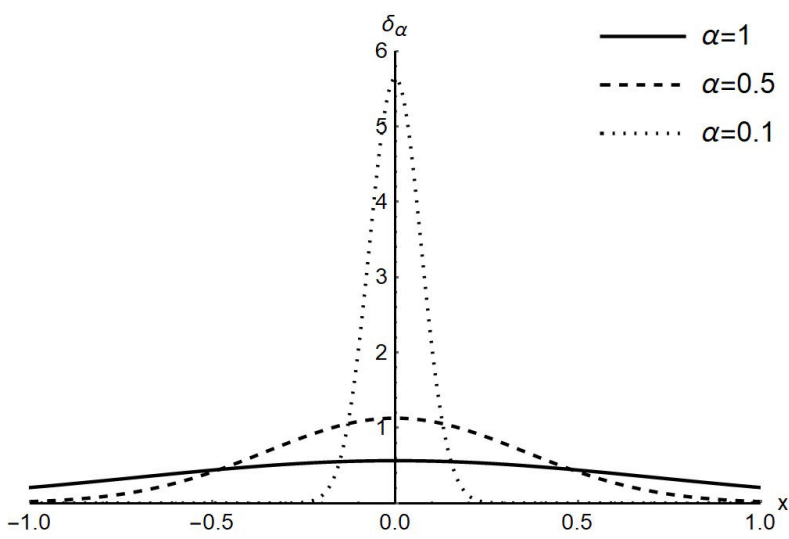

FIGURE 3. The function $\delta_{\alpha}(x)$ for $\alpha=1.0,0.5$ and 0.1 .

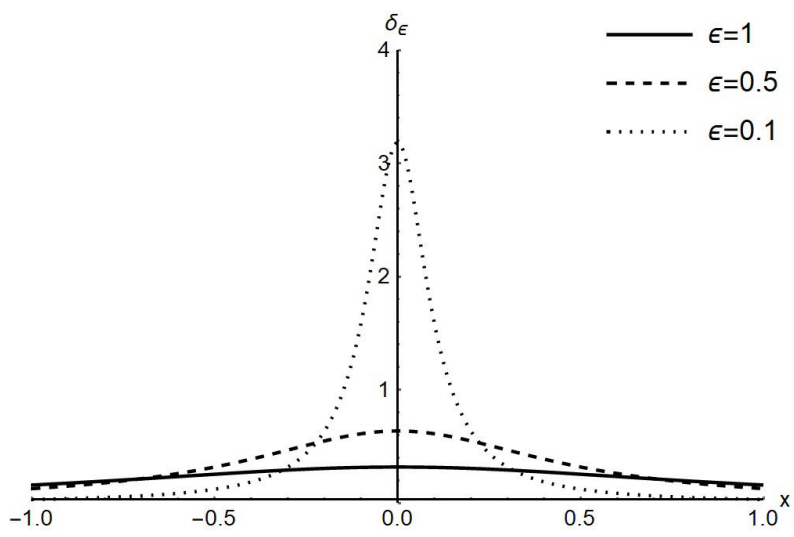

Figure 4. The function $\delta_{\varepsilon}(x)$ for $\varepsilon=1.0,0.5$ and 0.1 .

Using

$$
\frac{\varepsilon}{\pi} \int_{-\infty}^{+\infty} \frac{d x}{x^{2}+\varepsilon^{2}}=\frac{\varepsilon}{\pi} \int_{-\theta / 2}^{+\theta / 2} \frac{d \theta}{\varepsilon}=1
$$

where $x=\varepsilon \tan \theta$, we can follow the previous approach to verify the desired properties. In Fig. 4 the function $\delta_{\varepsilon}(x)$ is shown.

This representation of the delta function is slightly different from the preceding one. Defin the function $\delta_{n}(x)$ by

$$
\delta_{n}(x)=\left\{\begin{array}{c}
C_{n}\left(1-x^{2}\right)^{n} \text { for } 0 \leq|x| \leq 1 \\
0 \text { for }|x|>1
\end{array}\right.
$$

where the normalization constant $C_{n}$ is define so that $\int_{-1}^{1} \delta_{n}(x) d x=1$. After some algebra we have

$$
C_{n}=\frac{(2 n+1) !}{2^{2 n+1}(n !)^{2}} \text {. }
$$

If we know the behavior of the $C_{n}$ as $n \rightarrow \infty$ we have $\lim _{n \rightarrow \infty} \delta_{n}(x)=\delta(x)$ (see Fig. 5).

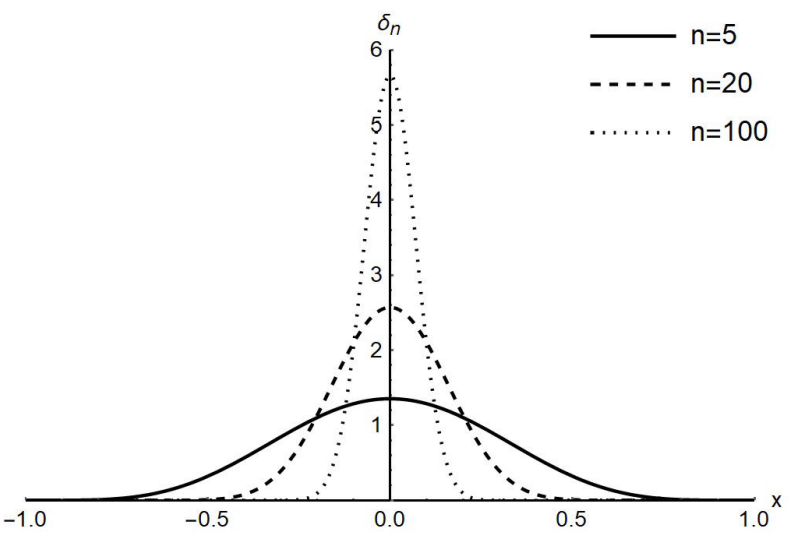

FIGURE 5. The function $\delta_{n}(x)$ for $n=5,20$ and 100 .

\section{A Physicist's approach of Dirac delta func- tion}

Consider the function

$$
f\left(x, x^{\prime}\right)=\frac{\sin a\left(x, x^{\prime}\right)}{\pi\left(x, x^{\prime}\right)}=\frac{a}{\pi}\left(\frac{\sin a\left(x, x^{\prime}\right)}{a\left(x, x^{\prime}\right)}\right)
$$

A plot of this for $a=10$ is shown in Fig. 6 below. Since $\lim _{\theta \rightarrow 0} \sin \theta / \theta=1$, we see that $f\left(x, x^{\prime}\right)$ has height $a / \pi$, an firs crosses the $x-x^{\prime}$ axis at $x-x^{\prime}=\pi / a$. Thus the area of the central peak is approximated by the area of the triangle with height $a / \pi$ and base $2 \pi / a$ for an area of $(1 / 2)(2 \pi / a)(a / \pi)=1$ which is independent of $a$. This underestimates the area under the main peak but ignores the tails, and these cancel each other out. Therefore, as $a \rightarrow \infty$ the height of $f\left(x, x^{\prime}\right)$ becomes infinit as its width goes to zero, while the area under the curve remains equal to 1 . More generally, the physicist's approach of the delta function is the limit

$$
\delta(x)=\lim _{\nu \rightarrow \infty} \frac{\sin \nu x}{\pi x}
$$

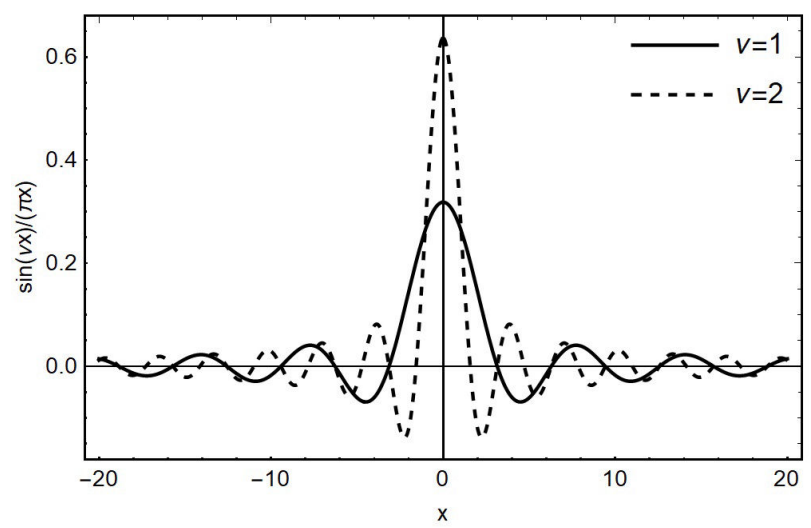

FIgURE 6. Delta function as the limit of the function $\sin \nu x / \pi x$ for $\nu=1$ and 2 . 


\section{Dirac delta with some applications in classi- cal electrodynamics}

\subsection{Charge density of a hydrogen atom}

The time-averaged potential of a neutral hydrogen atom is given by

$$
\phi(r)=\frac{q}{4 \pi \varepsilon_{0}} \frac{e^{-\alpha r}}{r}\left(1+\frac{\alpha r}{2}\right),
$$

where $q$ is the magnitude of the electronic charge, and $\alpha^{-1}=$ $a_{0} / 2 ; a_{0}$ being the Bohr radius.

Since this potential falls off faster than $1 / r$, it is reasonable to suspect that the total charge described by this potential is zero. We will fin the distribution of charge (both continuous and discrete) that will give this potential and interpret our result physically. Theoretically, we could just use Poisson's equation to fin the charge density; for $r>0$ is

$$
\rho(r)=-\varepsilon_{0} \nabla^{2} \phi(r),
$$

where

$$
\nabla^{2}=\frac{1}{r^{2}} \frac{\partial}{\partial r}\left(r^{2} \frac{\partial}{\partial r}\right),
$$

is Laplacian in spherical coordinates. We must be careful because of the singular behavior at $r=0$. For the case of $r \rightarrow 0$

$$
\lim _{r \rightarrow 0} \phi(r)=\lim _{r \rightarrow 0} \frac{q}{4 \pi \varepsilon_{0} r} .
$$

The singular nature of the Laplacian of $1 / r$ can be exhibited formally in terms of a Dirac delta function [16]:

$$
\nabla^{2}\left(\frac{1}{r}\right)=-4 \pi \delta(r)
$$

Combining Eqs. (22), (23) and Poisson's equation, we have got for $r \rightarrow 0$

$$
\rho(r)=q \delta(r) .
$$

We can multiply the right side of solution (24) by $e^{-\alpha r}$ without consequences. Away from the origin, $1 / r$ does not blow up and derivatives can be evaluated normally. This allows for a more elegant way of writing the discrete and the continuous parts together for all $r$ :

$$
\rho(r)=q \delta(r)-\frac{q \alpha^{3}}{8 \pi} e^{-\alpha r} .
$$

Obviously, the firs discrete term represents a positive point charge at the origin (proton) with one unit of elementary charge $q$, while second term corresponds to the negative electron with charge $-q$ that orbits around the proton. The continuous part of the charge density is more a statistical distribution of the location of the electron (negative electron cloud). This is of course only useful for conceptualization purposes, because at atomic size the system behaves quantum mechanically, not classically.

\subsection{Charge distribution as three-dimensional charge density}

Using Dirac delta function in appropriate coordinates, it is possible to express the charged distributions as a threedimensional charge density $\rho(\mathbf{r})$ in many of the problems of classical electrodynamics. For example, if we have a total charge $Q$ distributed over a thin spherical shell of radius $R$, in order to fin the charge distribution, it is required to use three-dimensional Dirac delta function that is define by Eq. (11). In this case the charge density should be in the form $\rho \propto \delta(r-R)$ because the charge distribution is zero except on a thin shell when $r$ equals $R$. The delta function insures that the charge density vanishes everywhere except when $r=R$, the radius of the sphere. Integrating $\rho$ over that shell, we should get $Q$ for the total charge:

$$
\int \rho(r) d V=\int A \delta(r-R) d V=Q,
$$

where $A$ is a constant that to be determined. Evaluate the integral and solve for $A$ :

$$
\begin{aligned}
\int A \delta(r-R) d V & =\int A \delta(r-R) \\
& \times r^{2} d(\cos \theta) d \phi d r=4 \pi R^{2} A=Q .
\end{aligned}
$$

So $A=Q / 4 \pi R^{2}$, can be found is charge distribution in the following form

$$
\rho(\mathbf{r})=\frac{Q}{4 \pi R^{2}} \delta(r-R) .
$$

\subsection{Volume charge density distribution for a group of $\mathbf{N}$ point charges}

A single point charge $q$ located at a point A characterized by the position vector $\mathbf{r}^{\prime}$ (Fig. 7). It can be represented by a continuous distribution [7]

$$
\rho(\mathbf{r})=q \delta\left(\mathbf{r}-\mathbf{r}^{\prime}\right),
$$

where $\delta\left(\mathbf{r}-\mathbf{r}^{\prime}\right)$ is three-dimensional delta function of argument $\mathbf{r}-\mathbf{r}^{\prime}$.

The integral of $\rho(\mathbf{r})$ overall space yields the charge $q$ :

$$
\int \rho(\mathbf{r}) d^{3} \mathbf{r}=\int q \delta\left(\mathbf{r}-\mathbf{r}^{\prime}\right) d^{3} \mathbf{r}=q .
$$

By virtue of the propriety of the three-dimensional delta function. The volume charge density distribution for a group $n$ point charges $q_{1}, q_{2}, \ldots, q_{N}$ located at $r_{1}^{\prime}, r_{2}^{\prime}, \ldots, r_{N}^{\prime}$ is given by

$$
\rho(\mathbf{r})=\sum_{i=1}^{N} q_{i} \delta\left(\mathbf{r}-\mathbf{r}_{i}^{\prime}\right) .
$$




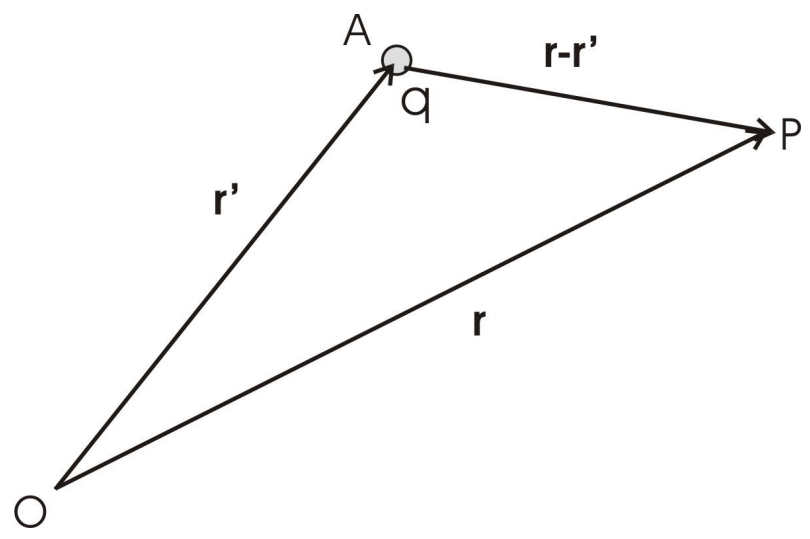

FIGURE 7. A single point charge.

\subsection{Volume charge density for a point dipole}

A single point electric dipole with dipole moment $\mathbf{p}$ that is located at a point A is shown in Fig. 8. The vector $\mathbf{r}^{\prime}$ is position vector with respect to an arbitrary point $\mathrm{O}$. A point electric dipole consisting of a two charges $-q$ and $+q$ a distance $\Delta r^{\prime}$ apart and laying in a direction characterized by the vector $\Delta \mathbf{r}^{\prime}$.

The point dipole is obtained in the limit when $q \rightarrow \infty$, while $\Delta r^{\prime} \rightarrow 0$ but in such a way that the product $q \Delta r^{\prime}$ remains finite Thus, the electric dipole moment is define by [8]

$$
\mathbf{p}=\lim _{\substack{q \rightarrow \infty \\ \Delta r^{\prime} \rightarrow 0}} q \Delta \mathbf{r}^{\prime}
$$

The total charge distribution is described by the density

$$
\rho(\mathbf{r})=q\left(\delta\left[\mathbf{r}-\left\{\mathbf{r}+\Delta \mathbf{r}^{\prime}\right\}\right]-\delta\left[\mathbf{r}-\mathbf{r}^{\prime}\right]\right),
$$

where firs part is corresponding volume density of charge $+q$, while the second part represents the density of point charge $-q$. Since the Dirac delta function is an even function of its argument, we fin

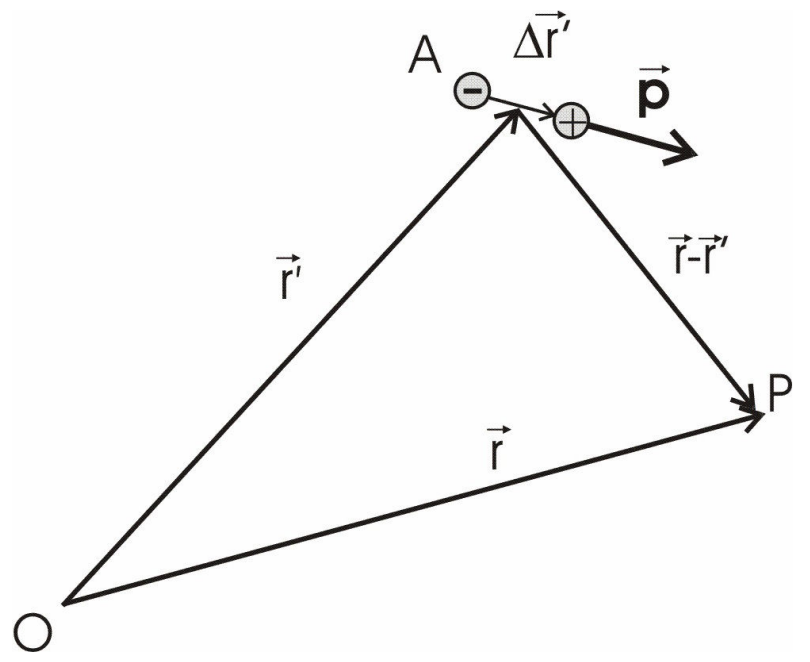

FIGURE 8. A point electric dipole.

$$
\rho(\mathbf{r})=q\left(\delta\left[\mathbf{r}^{\prime}-\mathbf{r}+\Delta \mathbf{r}^{\prime}\right]-\delta\left[\mathbf{r}^{\prime}-\mathbf{r}\right]\right)
$$

Using the Taylor expansion on the function (34), and after straightforward algebra we obtain

$$
\rho(\mathbf{r})=-\mathbf{p} \cdot \nabla \delta\left(\mathbf{r}^{\prime}-\mathbf{r}\right)
$$

Thus, a charge density of a point electric dipole can be expressed in terms of a highly singular vector, the gradient of delta function.

\subsection{The model of magnetic current}

Electron waves on a plasma guide attracted the attention of investigators for a long time. A comprehensive review on this topic can be found in [18]. A simplifie model of magnetic current in which the plasma is created by fictitiou magnetic current (Fig. 9) was presented in [19].

The typical method to fin electromagnetic wave propagation along the plasma waveguide assumed begin with Maxwell's equations in a form adapted to our case

$$
\begin{aligned}
& \nabla \times \mathbf{H}=\varepsilon_{0} \varepsilon \frac{\partial \mathbf{E}}{\partial t}, \\
& \nabla \times \mathbf{E}=-\mu_{0} \frac{\partial \mathbf{H}}{\partial t}-\mathbf{J}_{m},
\end{aligned}
$$

where $\mathbf{J}_{m}$ is magnetic current density which in cylindrical coordinate system has only azimuthal component and in form of Dirac delta function is given as

$$
J_{m \phi}=U \delta(\rho-R) \delta(z)
$$

The adequate coordinate system here is a cylindrical system $(\rho, \phi, z)$; the $z$-axis coincides with the axis of the guide, whereas $\rho$ and $\phi$ are the corresponding radius and angle in the perpendicular plane. Therefore, the components of the electric fiel $\vec{E}$ are $\left(E_{p}, E_{\phi}, E_{z}\right)$ and magnetic fiel $\vec{H}$ $\left(H_{p}, H_{\phi}, H_{z}\right)$. Here the typical method for findin the solution of wave equation connected to the mentioned problem of electromagnetic wave propagation along the plasma waveguide was used $[19,20]$.

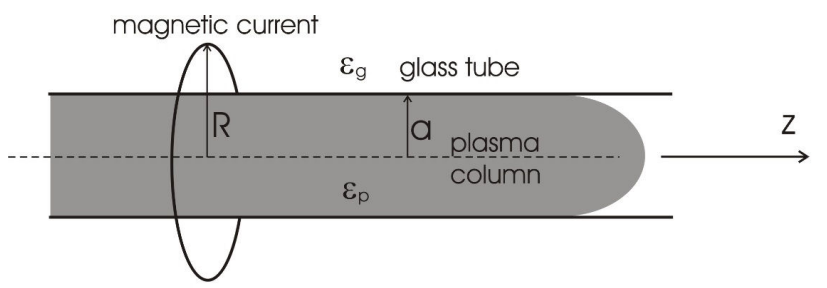

FIGURE 9. The plasma created by magnetic current. The radius of plasma column is $a$ whereby it is $R>a ; \varepsilon_{p}$ and $\varepsilon_{g}$ are plasma and glass permittivity, respectively. 


\section{Conclusion}

The study of Dirac delta function is now widely used in classical electrodynamics, quantum mechanics and applied mathematics. The Dirac delta function approach provides us with a unifie approach in treating discrete and continuous distributions. Our students of physics encountered a few issues when using or interpreting the Dirac delta function. This paper contributes to the limited body of research on student difficultie with the Dirac delta function by presenting its defini tion and properties. The paper framework provided an organizing structure for Dirac delta function analysis that helped us to identify nodes in students' work where key difficultie appeared. The method of using Dirac delta function to calculate charge distribution for the point charge as well as point electric dipole is presented. Such examples could provide a longitudinal perspective on the growth of student understanding of classical electrodynamics, allowing researchers and instructors to focus their efforts on addressing those difficultie that are most common throughout the physics curriculum. Thus, the Dirac delta function is one of the most essential and widely used concepts in physics and in mathematical analysis.

\section{Acknowledgments}

This work was supported by the Serbian Ministry of Education, Science and Technological Development (Agreement No. 451-03-9/2021-14/200122).
1. J. Lützen, The Prehistory of the Theory of Distributions (Springer-Verlag, New York, 1982), https://doi.org/ 10.1007/978-1-4613-9472-3

2. P. A. M. Dirac, The physical interpretation of quantum mechanics, Proc. R. Soc. Lond. A 180 (1942) 1, https : //doi. org/10.1098/rspa.1942.0023

3. P. A. M. Dirac, The Principles of Quantum Mechanics (Oxford University Press, Oxford, 1930).

4. L. Schwartz, Theórie des distributions (Hermann, Paris, 1952).

5. M. J. Lighthill, An Introduction to Fourier Analysis and Generalised Functions (Cambridge University Press, Cambridge, 1964), https://doi.org/10.1017/ CB09781139171427

6. C. P. Frahm, Some novel delta-function identities, Am. J. Phys. 51 (1983) 826, https : //doi.org/10.1119/1.13127

7. S. M. Blinder, Delta functions in spherical coordinates and how to avoid losing them: Fields of point charges and dipoles, $A m$. J. Phys. 71 (2003) 816, https: //doi.org/10.1119/1. 1566429

8. V. Namias, Application of the Dirac delta function to electric charge and multipole distributions, Am. J. Phys. 45 (1977) 624, https://doi.org/10.1119/1.10779

9. P. T. Leung and G. J. Ni, On the singularities of the electrostatic and magnetostatic dipole fields Eur. J. Phys. 27 (2006) N1, https://doi.org/10.1088/0143-0807/27/4/ N01.

10. P. R. Tangherlini, General relativistic approach to the Poincaré compensating stresses for the classical point elec- tron, Nuovo Cim. 26 (1962) 497, https : // doi.org/10. $1007 / \mathrm{BF} 02771821$

11. A. Gsponer, On the physical interpretation of singularities in Lanczos-Newman electrodynamics, arXiv:gr-qc/0405046.

12. A. Gsponer, The locally-conserved current density of the Lienard-Wiechert field, arXiv: arXiv:physics/0612090.

13. B. R. Wilcox and S. J. Pollock, Upper-division student difficul ties with the Dirac delta function, Phys. Rev. ST Phys. Educ. Res. 11 (2015) 010108, https://doi.org/10.1103/ PhysRevSTPER.11.010108

14. R. A. Brewster and J. D. Franson, Generalized delta functions and their use in quantum optics, J. Math. Phys. 59 (2018) 012102, https://doi.org/10.1063/1.4985938

15. E. Parker, An apparent paradox concerning the fiel of an ideal dipole, Eur. J. Phys. 38 (2017) 025205, https : / / doi .org/ 10.1088/1361-6404/aa55a6.

16. J. D. Jackson, Classical Electrodynamics, 3rd ed. (Wiley, New York, 1999), pp. 34-35.

17. D. J. Griffiths Introduction to Electrodynamics (Prentice Hall, New Jersey, 1999).

18. V. Babović, Excitation of Surface Electron Plasma Waves (University of Kragujevac, Kragujevac, 1999).

19. M. S. Kovačević, M.Sc. thesis, University of Belgrade, 2000.

20. M. S. Kovačević, L. Kuzmanović, M. M. Milošević, and A. Djordjevich, An estimation of the axial structure of surfacewave produced plasma column, Phys. Plasmas 28 (2021) 023502, https://doi.org/10.1063/5.0035035 